\title{
Distinct spatial frequency sensitivities for processing faces and emotional expressions
}

\author{
Patrik Vuilleumier ${ }^{1,2}$, Jorge L Armony ${ }^{2,3}$, Jon Driver ${ }^{2}$ \& Raymond J Dolan ${ }^{4}$ \\ High and low spatial frequency information in visual images is processed by distinct neural channels. Using event-related \\ functional magnetic resonance imaging ( $F M R I)$ in humans, we show dissociable roles of such visual channels for processing faces \\ and emotional fearful expressions. Neural responses in fusiform cortex, and effects of repeating the same face identity upon \\ fusiform activity, were greater with intact or high-spatial-frequency face stimuli than with low-frequency faces, regardless of \\ emotional expression. In contrast, amygdala responses to fearful expressions were greater for intact or low-frequency faces than for \\ high-frequency faces. An activation of pulvinar and superior colliculus by fearful expressions occurred specifically with low- \\ frequency faces, suggesting that these subcortical pathways may provide coarse fear-related inputs to the amygdala.
}

Specialized neural systems have evolved for processing stimuli with particular emotional significance. Expedient detection of fear-related cues is critical for survival, and relies on dedicated circuits in which the amygdala has a key role ${ }^{1}$. In humans, the amygdala is consistently activated by fearful faces, even when those faces are not consciously perceived $^{2,3}$. In some situations, the amygdala may also respond to fearful faces presented outside the focus of attention and not explicit ly identified, despite the fact that inattention is associated with decreased activation in face-selective areas in fusiform cortex ${ }^{4}$

It has been proposed that the amygdala may receive direct subcortical inputs from the thalamus to enable crude but fast signals about fear-related stimuli to bypass slower cortical analysis in ventral visual pathways ${ }^{1}$. Specifically, a retinal-collicular-pulvinar pathway has been proposed $^{5,6}$. This subcortical visual system, phylogenetically older than the cortical visual system of primates, is capable of mediating some residual unconscious visual processing after destruction of cortical pathways ${ }^{7}$. To date, however, only a few anatomical studies in nonprimate animals ${ }^{1}$, plus indirect evidence from humans $s^{5,6,8}$, support a subcortical route to the amygdala for processing fear-related stimuli.

We used face stimuli with different spatial frequencies to investigate whether the amygdala and ventral visual cortex have different inputs in the normal human brain. We exploited the fact that distinct neural pathways in the visual system are selectively sensitive to different ranges of spatial frequencies. Higher spatial frequency (HSF) information projects chiefly to the ventral cortical visual stream via parvocellular channels ${ }^{9,10}$ with fine resolution but slow responses. Conversely, the dorsal parietal stream and connected subcortical regions, such as superior colliculus and pulvinar, are tuned for lower spatial frequency (LSF) information that is received via magnocellular channels ${ }^{11-13}$. This latter pathway might provide rapid, but coarse, visual signals to the amygdala. Some magnocellular inputs also reach striate and extrastriate cortex but contribute less to major ventral visual function. Subcortical pathways do not receive parvocellular inputs ${ }^{9,10}$.

Our event-related fMRI study addressed two main questions by decomposing face stimuli into their HSF or LSF components (Fig. 1). First, we tested whether processing in ventral visual cortex (faces) and the amygdala (fearful expressions) are differentially sensitive to HSF versus LSF inputs. Second, we assessed whether facial information in HSF and LSF ranges might proceed via distinct cortical or subcortical pathways. Psychophysical studies in human observers suggest that distinct spatial frequency ranges may have different roles in face processing ${ }^{14-18}$. LSF components (2-8 cycles/face) convey global configurational information ${ }^{14,15}$, sufficient to provide only coarse emotional cues ${ }^{16,19}$. HSF components ( $8-16$ cycles/face), in contrast, convey fine-grained information important for precise recognition of identity ${ }^{14,18,20-22}$ and for more detailed analysis of facial traits, such as age or expression-related wrinkles ${ }^{16,23}$.

On each trial, during whole-brain scanning, our participants judged the gender of a face that showed either a fearful or neutral expression and had one of three spatial frequency contents: an intact broad spatial frequency (BSF), HSF alone or LSF alone (Fig. 1). Different stimulus types were presented in random order, with emotional expression irrelevant to the gender task. Gender judgments rely equally on HSF and LSF information, with no dominance by either range ${ }^{16}$. Our fMRI results were consistent with the hypothesis that brain regions such as the amygdala, which are normally responsive to fearful faces, might be 'blind' to fear in the HSF faces if they primarily receive subcortical and magnocellular LSF inputs. In contrast, brain regions such as ventral visual cortex, which receive substantial parvocellular inputs, responded more to HSF than LSF face stimuli. We also found that repeating facial identity, irrespective of expression, produced distinct effects in visual cortex as a function of spatial frequency content .

${ }^{1}$ Laboratory for Neurology and Imaging of Cognition, Department of Clinical Neuroscience \& Physiology, University of Geneva Medical Center, 1 Michel-Servet, 1211 Geneva, Switzerland. ${ }^{2}$ Institute of Cognitive Neuroscience, University College London, 17 Queen Square, London WC1N 3AR, UK. ${ }^{3}$ Douglas Hospital Research Centre, McGill University, 6875, BIvd. LaSalle, Verdun, Québec H4H 1R3, Canada. "Wellcome Department of Imaging Neuroscience, University College London, 12 Queen Square, London WC1N 3BG, UK. Correspondence should be addressed to P.V. (patrik.vuilleumier@medecine.unige.ch). 
Figure 1 Example stimuli. Faces with a normal (intact) broad spatial frequency (BSF) content (left column) were filtered to contain only a high range or low range of spatial frequencies (HSF or SLF; middle and right columns respectively). Six possible face types were equally distributed in a $2 \times 3$ factorial design ( $n=40$ per cell), with either a fearful or neutral expression (half each), and either a BSF, LSF or HSF content (all shown in random order; duration 200 ms each; mean interstimulus interval $6.1 \mathrm{~s}$ ). Individual face stimuli were counterbalanced across participants, with different expressions and spatial frequency contents chosen for each face across participants. In addition, each individual was repeated once (after a randomly chosen lag of between 25 and 35 intervening stimuli) with either the same or a different spatial frequency content (that is, same or different image of the same person; $n=20$ faces for each type of repetition; see Results).

\section{RESULTS}

Behavioral performance during scanning

We measured accuracy and reaction times for the gender discrimination task in each participant. Errors were more frequent for HSF and LSF faces than for intact BSF faces (mean $6.4,7.2$ and $1.7 \%$, respectively; $F_{2,24}=6.32$,
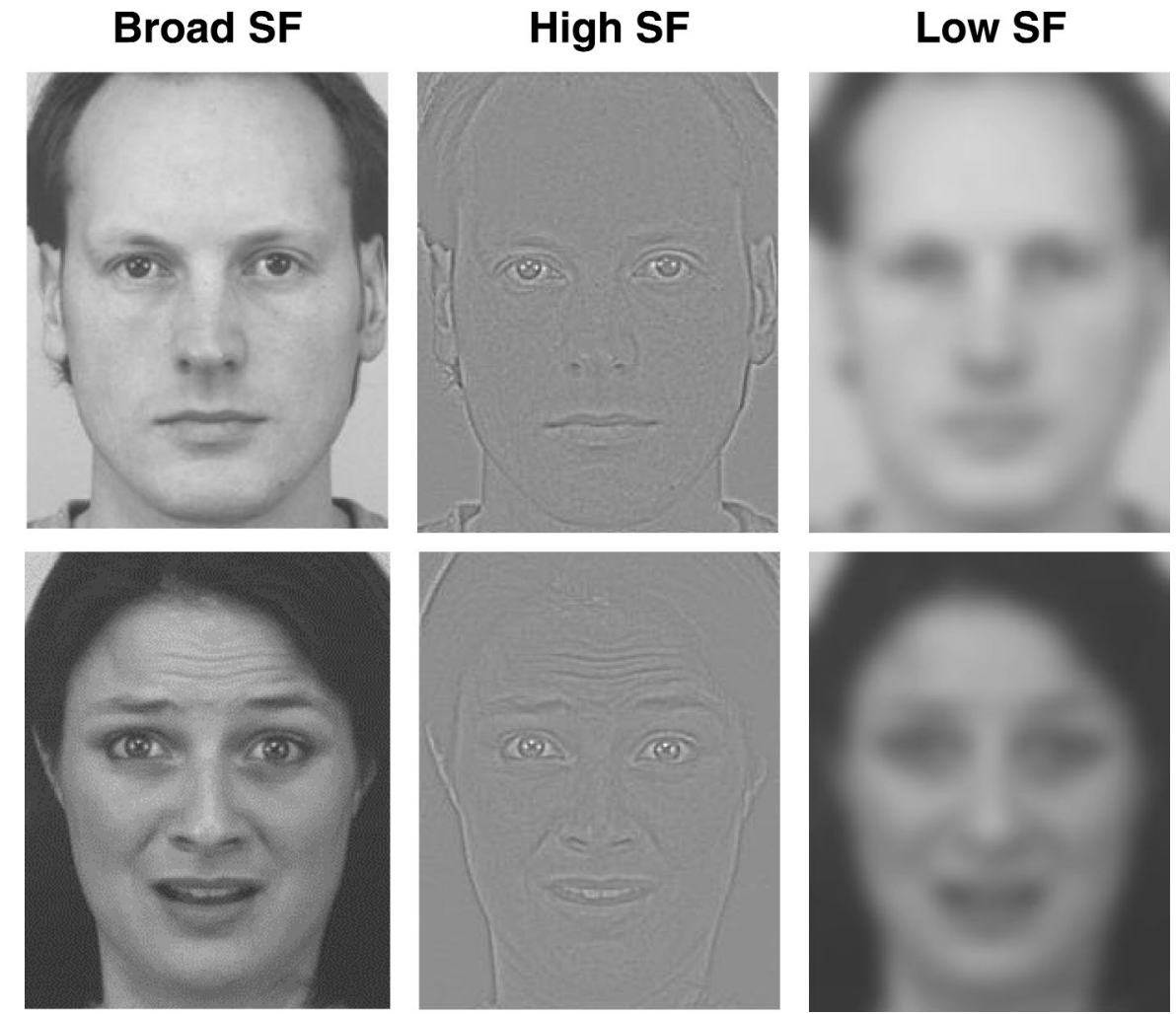
$P=0.006)$. Similarly, correct responses were slower to both HSF and LSF faces than to intact BSF faces $\left(882,837\right.$ and $802 \mathrm{~ms}$, respectively; $F_{2,24}=7.58$, $P=0.003)$. Importantly, there was no difference between HSF and LSF for accuracy $\left(t_{25}=0.46, P=0.65\right)$ or reaction time (RT; $t_{25}=1.62$, $P=0.12$ ); and there was no significant main effect or interaction involving emotional expression for either measure. Repeating the same face identity across different images produced no significant effect on RT or accuracy (consistent with tenuous behavioral repetition-priming in gender-judgment tasks using unfamiliar faces ${ }^{24}$ ).

\section{Imaging data}

Functional images were analyzed by statistical parametric mapping (SPM99) using the general linear model applied at each voxel across

Table 1 Effects of spatial frequency content

\begin{tabular}{llrrrrr}
\hline Side & Area & $\boldsymbol{x}$ & $\boldsymbol{y}$ & $\boldsymbol{z}$ & $\boldsymbol{t}$ \\
$\mathrm{HSF}>\mathrm{LSF}$ faces (regardless of expression) & & & & \\
\hline $\mathrm{L}$ & fusiform gyrus & -34 & -62 & -18 & 5.28 & \\
$\mathrm{~L}$ & inferior temporal gyrus & -20 & -88 & -14 & 5.08 & \\
$\mathrm{~L}$ & inferior occipital gyrus & -8 & -94 & -4 & 3.44 & * \\
$\mathrm{R}$ & fusiform gyrus & 32 & -62 & -12 & 2.73 & ** \\
$\mathrm{R}$ & inferior occipital gyrus & 24 & -98 & -6 & 4.45 & \\
$\mathrm{R}$ & lateral occipital gyrus & 34 & -76 & 4 & 3.26 & * \\
$\mathrm{LSF}>$ HSF faces (regardless of expression) & & & & \\
\hline $\mathrm{L}$ & parieto-occipital cortex & -38 & -72 & 22 & 4.11 & \\
$\mathrm{~L}$ & angular gyrus & -48 & -64 & 28 & 3.71 & \\
$\mathrm{R}$ & parieto-occipital cortex & 52 & -56 & 4 & 5.23 & \\
$\mathrm{R}$ & retrosplenial cortex & 10 & -52 & 14 & 4.45 & \\
\hline
\end{tabular}

All peaks $P<0.001$ uncorrected (random-effect analysis), except ${ }^{*} P<0.005$,

${ }^{* *} P<0.01$. the whole brain. We first determined areas showing a main effect of different frequency inputs, comparing neural responses evoked by LSF or HSF faces regardless of expression. A greater response to HSF than LSF stimuli (HiNeut + HiFear $>$ LoNeut + LoFear) was observed in bilateral fusiform and inferior temporo-occipital cortex (Table 1 and Fig. 2a,b), particularly in the left hemisphere ${ }^{14}$. The opposite contrast (LoNeut + LoFear $>$ HiNeut + HiFear) revealed a greater response to LSF than HSF stimuli in bilateral parieto-occipital and retrosplenial cortex (Table 1). These differential effects are consistent with proposals ${ }^{9,10}$ that HSF inputs project more to ventral extrastriate areas that subserve fine perceptual shape-analysis, whereas LSF inputs project more to the dorsal visual stream. Fusiform cortex in the ventral stream is critically involved in face processing ${ }^{4,25}$. Our findings show that fusiform cortex was equally activated by intact and HSF faces (Fig. 2c), both of which allow for detailed face perception and recognition of identity ${ }^{14,18,21}$. This region was generally less sensitive to LSF stimuli, which convey less distinctive facial traits ${ }^{15,21,22}$, and this was found irrespective of facial expression. These differential effects of spatial frequency in left fusiform were confirmed by analysis of individual subject data (Fig. 3a). See Supplementary Fig. 1 online for right fusiform data and analyses based on individual subjects' maxima, rather than group analyses.

We found further evidence of spatial-frequency selectivity in face processing when we examined effects of repeating the same person's face. We tested for 'repetition-priming' of the fMRI response ${ }^{26}$ that differed according to whether the initial trace was induced by exposure to a HSF or LSF face stimulus. Previous fMRI studies report selective decreases in the response of regions tuned to a particular stimulus type when 'primed' by previous exposure of the same or a related stimulus ${ }^{27}$, hence revealing stimulus properties selectively extracted by regional neurons ${ }^{26}$. In the present study, the face of any particular person was presented only twice, using either the same or a 
a

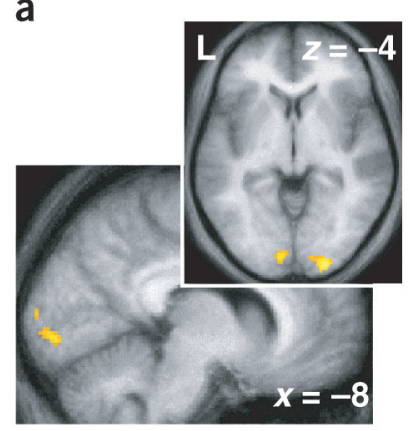

b

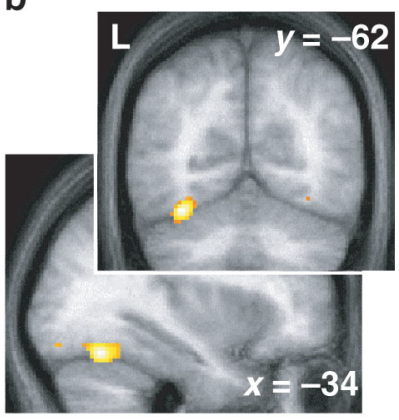

C

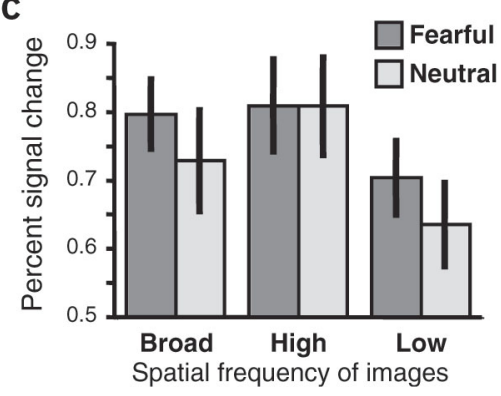

Figure 2 Activation in extrastriate visual cortex, on the mean anatomical brain scan of participants. Occipital (a) and posterior fusiform (b) areas in ventral temporal cortex where activity was greater for HSF than LSF faces, irrespective of expression. (c) Average activity ( \pm s.e.m.) across conditions for the left fusiform cluster (mean $x y z=-36-54-16,186$ voxels at $P<0.01$ ) as shown in $\mathbf{b}$, demonstrating greater responses to either BSF or HSF than LSF stimuli. A similar pattern was found in the right hemisphere (Table 1) and in face-responsive fusiform areas defined individually for each subject, rather than at a group level (Supplementary Fig. 1).

different image for the repetition. Intact BSF faces were repeated in the same intact format (mean lag, 28 intervening stimuli), whereas faces presented initially in HSF format were later repeated in LSF (mean lag 29) and vice versa (mean lag 29; no lag difference between conditions, $F_{1,12}=0.11$ ). This enabled us to test for brain regions that encode lasting facial-identity information that transfers across images of entirely different spatial frequency.

A comparison of fMRI responses to the first versus second presentation of all faces, irrespective of spatial frequency content and expression, revealed a main effect of repetition-related decreases in bilateral fusiform cortex (Table 2 and Fig. 4a,b). This reduction was also evident for repetition of intact BSF stimuli considered alone (right anterior and posterior fusiform: peak coordinates $(x y z), 36-42-12, t=5.16$;

Figure 3 Differential effects of spatial frequency in individual subjects. Mean parameter estimates of activity for HSF and LSF faces are shown for (a) left fusiform cortex and (b) amygdala in all 13 participants, extracted from a 10-mm region of interest centered on the group maxima for the left fusiform cortex (-34-62-18) or right amygdala (20 $-10-30)$. Circles correspond to individual subject data. Responses were consistently greater for HSF than LSF faces irrespective of expression in the fusiform, whereas responses to fear were consistently greater for LSF than HSF faces in amygdala.
$42-36-24, t=4.91 ; 52-56-22, t=3.81$; left anterior and posterior fusiform: $-46-38-14, t=3.97 ;-32-64-22, t=4.43$; all $P \leq 0.001$ ). Significant repetition decreases were also evident in fusiform cortex for LSF faces that had previously been seen in HSF, as compared to new faces seen in LSF (Table 2 and Fig. 4c). However, no such repetition decreases were found for faces repeated in HSF after initial exposure in LSF. Direct tests for an interaction between repetition and spatial frequency (that is, greater decreases for faces repeated after an initial HSF exposure than for faces repeated after an initial LSF exposure) again identified ventral temporal areas, with the most prominent peaks in anterior fusiform regions bilaterally (Fig. 4b), plus right parietal cortex and insula (Table 2). No voxel showed an opposite interaction.

Taken together, the above results suggest that visual recognition mechanisms for processing faces in ventral extrastriate cortex are tuned to encode predominantly HSF information. In addition, our data indicate that the trace of previous exposures to an identity producing a subsequent repetition-priming effect on neural responses across different images may also show HSF predominance (in the initial laying down of the trace). This accords with the fact that although a wide range of spatial frequencies, both low and high, may reach ventral visual pathways ${ }^{10}$ to allow perception of both facial configuration and details ${ }^{14,15,17}$, the finer HSF cues conveyed by parvocellular inputs might have a predominant role in establishing long-term representations in temporal cortex ${ }^{9}$ and in identifying faces ${ }^{14,18,21}$. Stored traces derived from a previous exposure to fine-grained HSF information may facilitate subsequent processing of impoverished (or LSF) stim$\mathrm{uli}^{28}$. This could result in greater generalization across different image inputs than does prior exposure to LSF stimuli, especially in anterior fusiform areas that may encode abstract face identity ${ }^{29}$.

In contrast to the predominant role of the HSF range for faceprocessing in ventral visual cortex, we found that the LSF range has a crucial role in the processing of emotional fearful expression, particularly for the amygdala and subcortical pathways. Brain areas specifically engaged by fear were first determined from the main effect of emotion across all stimulus types (BrFear + HiFear + LoFear $>$ BrNeut + LoNeut + HiNeut). As expected, this revealed significant activation in bilateral amygdala (Fig. 5a) as well as fusiform, insula, retrosplenial cortex and temporal regions (Table 3), all previously implicated in emotional processing ${ }^{4,30}$. Critically, when examining the effect of emotion for the images of different spatial frequency (Fig. 5d), a significant response to fear was observed in bilateral amygdala for LSF faces considered alone (right peak $x y z, 16-2-28$, $t=4.09, P=0.001$; left peak $x y z,-16-8-22, t=3.02, P=0.005)$, as
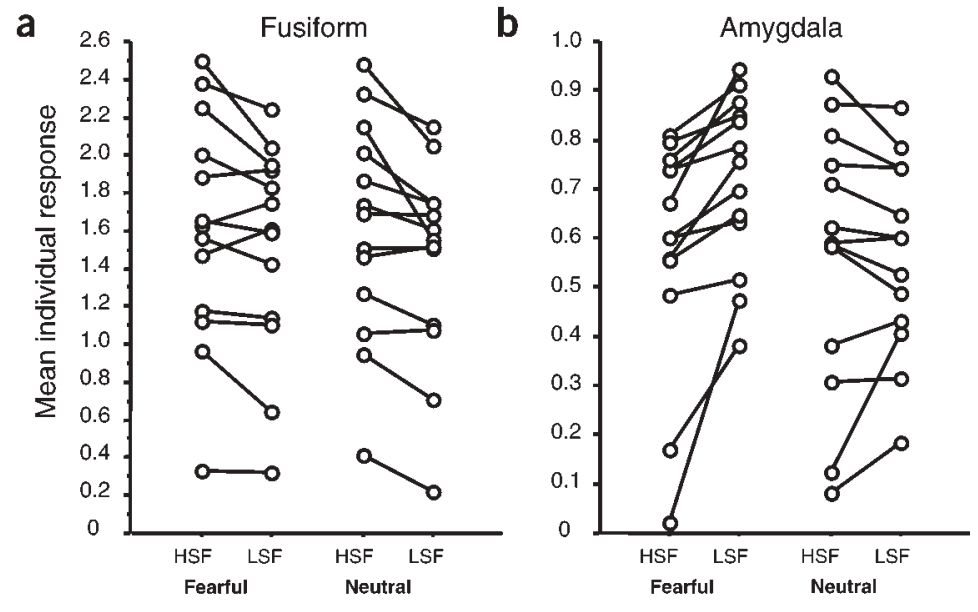
Table 2 Effects of repetition priming

\begin{tabular}{lrrrrl}
\hline Side & Area & $\boldsymbol{x}$ & $\boldsymbol{y}$ & $\boldsymbol{z}$ & \multicolumn{1}{l}{$\boldsymbol{t}$} \\
For all faces (collapsed across all & spatial & frequency formats) & \\
\hline $\mathrm{L}$ & fusiform gyrus (post.) & -44 & -66 & -24 & 5.01 \\
& (post.) & -36 & -56 & -12 & 4.06 \\
$\mathrm{~L}$ & lateral occipital gyrus & -38 & -80 & 4 & 6.45 \\
$\mathrm{R}$ & fusiform gyrus (post.) & 54 & -62 & -20 & 4.61 \\
& (post.) & 36 & -68 & -8 & 4.53 \\
$\mathrm{R}$ & (ant.) & 42 & -36 & -24 & 4.88 \\
$\mathrm{R}$ & inferior frontal gyrus & 44 & 8 & 16 & 6.05 \\
$\mathrm{~L}$ & precentral frontal gyrus & -34 & -6 & 66 & 4.93 \\
$\mathrm{~L}$ & inferior parietal lobule & -60 & -58 & 20 & 5.14 \\
$\mathrm{R}$ & inferior parietal lobule & 66 & -30 & 26 & 5.17 \\
$\mathrm{R}$ & superior parietal lobule & 40 & -32 & 38 & 5.08
\end{tabular}

LSF faces primed by HSF (LSF first > LSF repeated after HSF)

\begin{tabular}{rrrrrr}
\hline $\mathrm{L} \quad$ fusiform gyrus (post.) & -42 & -66 & -16 & 4.36 \\
& (post.) & -48 & -58 & -8 & 4.06 \\
& fusiform gyrus (ant.) & -36 & -28 & -20 & 4.26 \\
& (ant.) & -32 & -36 & -26 & 4.18 \\
$\mathrm{R} \quad$ fusiform gyrus (ant.) & 30 & -32 & -30 & 5.39 \\
& (post.) & 44 & -50 & -22 & 3.2
\end{tabular} *

HSF primed by LSF (HSF first > HSF repeated after LSF)

$$
\text { n.s. }
$$

Repetition $\times$ frequency interaction (decrease for LSF primed by HSF $>$ HSF primed by LSF)

\begin{tabular}{rrrrrrr}
\hline L & fusiform gyrus (ant.) & -40 & -30 & -20 & 7.55 & \\
& (ant.) & -28 & -36 & -34 & 3.28 & * \\
& (post.) & -44 & -52 & -8 & 3.46 & * \\
$\mathrm{R}$ & fusiform gyrus (ant.) & 30 & -34 & -32 & 5.51 & \\
& (ant.) & 36 & -42 & -22 & 4.12 & \\
& (post.) & 50 & -46 & -18 & 3.59 & * \\
$\mathrm{R}$ & inferior parietal cortex & 50 & -16 & 24 & 5.33 & \\
$\mathrm{R}$ & insula & 46 & 14 & 4 & 5.13 & \\
\hline
\end{tabular}

All peaks $P<0.001$ uncorrected (random-effect analysis), except ${ }^{*} P<0.005$.

well as for intact BSF faces considered alone (left peak $x y z,-26-2$ $-32, t=5.85$; right peak $x y z, 30-4-28, t=4.12$, all $P \leq 0.001)$. By contrast, there was no significant amygdala response for HSF fearful versus neutral stimuli, even at liberal statistical thresholds (all $t<0.82$ at same amygdala peaks as above, all $P>0.20$ ). This lack of amygdala response for fear in HSF faces contrasts with this higher spatial frequency range producing greater activation in occipito-temporal cortices (Fig. 2; see also Fig. 3b for effects of fear in LSF versus HSF faces in individual subjects). It is also consistent with the idea that the amygdala may receive visual inputs that have some independence from face processing in ventral visual cortex. These inputs may depend on neural pathways preferentially tuned to LSF rather than HSF, in accord with proposals of a subcortical route carrying visual inputs to amygdala via magnocellular tecto-pulvinar pathways ${ }^{1,5}$.

Evidence for this proposal comes from our findings of brain responses that reflect a significant interaction between emotionality and spatial frequency range of the stimulus, that is, greater activation by fearful than neutral expression specifically for LSF rather than HSF
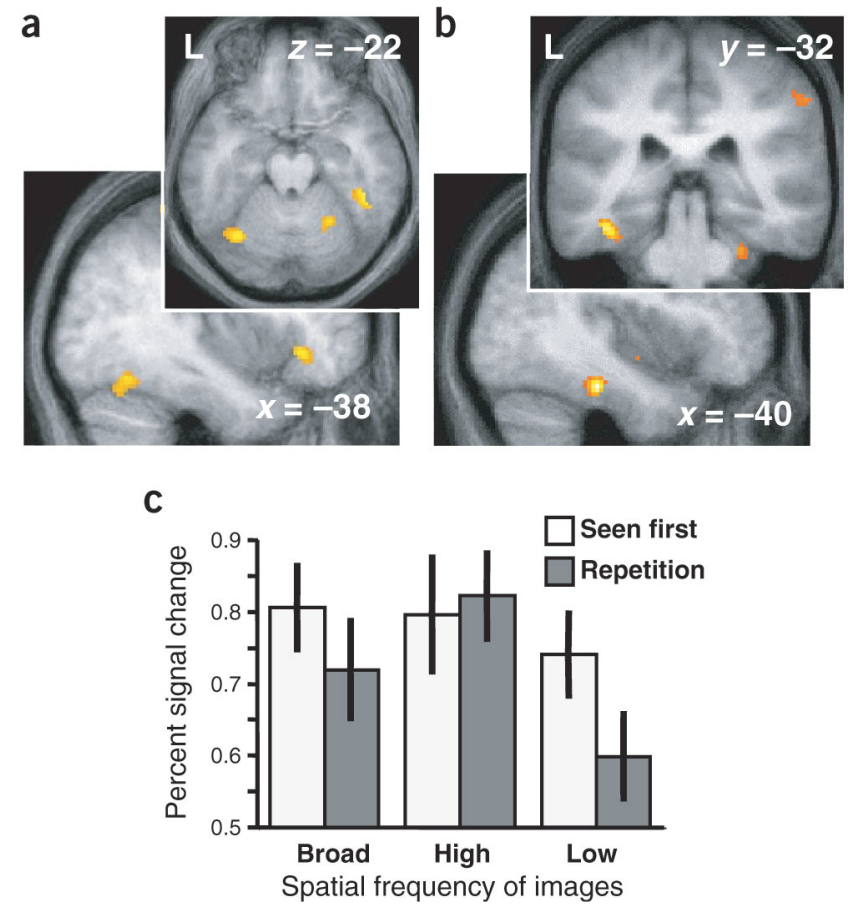

Figure 4 Effects of face identity repetition in extrastriate cortex, on the mean anatomical brain scan of participants. (a) The same posterior fusiform areas as shown in Fig. $\mathbf{2}$ and (b) more anterior fusiform regions showed significant repetition-related decreases. These ocurred for BSF faces repeated in the same BSF version (compared with BSF faces seen for the first time), or for faces repeated in LSF after exposure to HSF versions of the same individual (compared with LSF versions of faces seen for first time). But there were no priming decreases for faces repeated in HFS after initial LSF exposure. A significant repetition $x$ frequency interaction was also found in anterior fusiform (b), where priming effects were specifically greater for repeated stimuli initially shown in HSF. (c) Average activity ( \pm s.e.m.) across conditions for the same left fusiform cluster (mean $x y z,-36-54-16 ; 186$ voxels) as in Fig. 2, showing not only greater responses to either BSF or HSF than LSF stimuli, but also greater repetition-priming decreases after previous exposure to either BSF or HSF versions of the repeated face.

faces $([$ LoFear - LoNeut $]>$ [HiFear - HiNeut $])$. Such an interaction was found not only in the amygdala (Fig. 5 b and Table 3 ), but also in bilateral posterior-inferior thalamus, corresponding to the visual pulvinar nuclei ${ }^{31}$, and extending into the right superior colliculus (Fig. 5c,e and Table 3). Similar effects were observed in retrosplenial cortex, posterior cingulate and insula (Table 3), all connected not only with amygdala and limbic areas, but also with the pulvinar ${ }^{31}$. No voxel showed an opposite interaction of greater response to fearful expression in HSF compared to LSF faces.

The superior colliculus activation was not explained by differences in overt eye movements across the different types of stimuli, as measured on-line during scanning in each participant. Repeated-measure ANOVAs on mean eye-position data over a $200-\mathrm{ms}$ period after stimulus onset showed no significant effects or interaction due to spatial frequency, emotion or repetition priming. Moreover, stimulus duration (200 ms) was too brief for saccades to alter visual inputs during each display presentation.

We note that fusiform regions showed greater responses to fearful than neutral faces for LSF and BSF but not HSF faces (Fig. $2 \mathrm{c}$ and Table 3), despite the fact that these regions were more activated by 
a

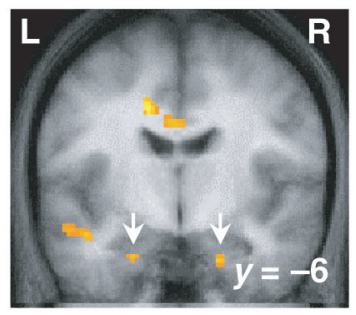

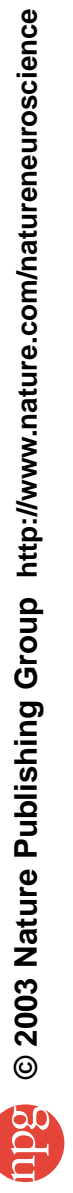

b

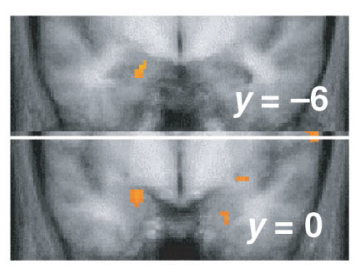

d

e
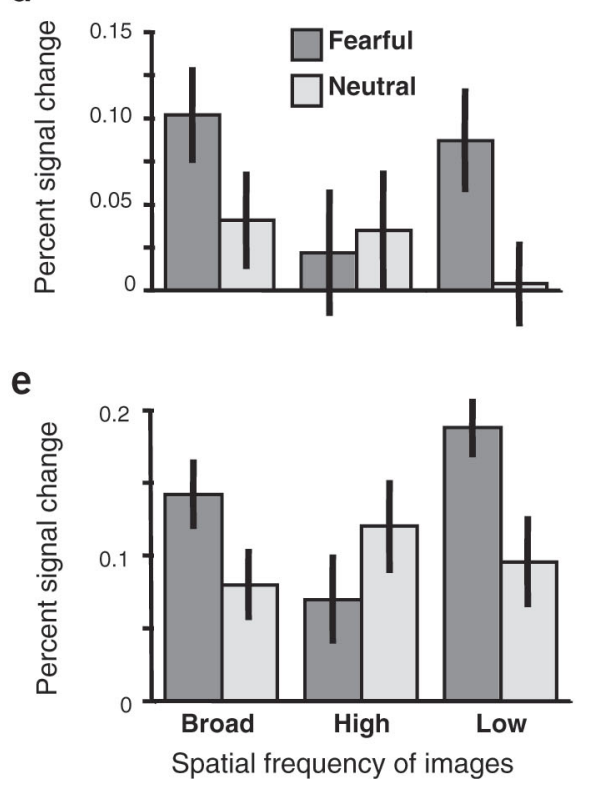

HSF information overall and showed more priming from an HSF trace. This may be consistent with emotional expression enhancing fusiform responses to faces via feedback influences received from amygdala $^{4,32}$, where emotion was evidently extracted better from LSF than HSF cues. Finally, whereas the fusiform showed stronger repetition effects from HSF traces than from LSF traces, the amygdala did not show any repetition effects in the present long-term priming paradigm for either case, even when considering only fearful faces.

\section{Behavioral ratings of facial expression}

To assess whether differential amygdala responses to fear as a function of spatial frequency might be an 'artifact' of a greater intensity of consciously perceived emotion in LSF faces, we asked a separate group of 12 healthy participants to rate the degree of emotion on a scale from zero (totally neutral) to 5 (extraordinarily fearful) for each of the intact, HSF and LSF faces (half fearful, half neutral). Their ratings showed a main effect of expression (mean, 3.4 for fearful versus 1.4
Figure 5 Activation in amygdala and subcortical pathways, on the mean anatomical brain scan of participants. (a) Bilateral amygdala response (vertical arrows) to fearful versus neutral faces, when collapsing over all spatial frequency ranges. An interaction of emotional expression $\times$ spatial frequency content was found in similar bilateral amygdala regions (b) and in bilateral posterior-inferior thalamus (c, see oblique arrows), with the latter extending ventrally into the superior colliculus on the right side. (d) Average activity ( \pm s.e.m.) across conditions from a left amygdala cluster (mean $x y z$, $-24-6-28 ; 8$ voxels at $P<0.01$ ) as depicted in a, that showed a significant response to fearful expression in both intact BSF faces and filtered LSF faces, but did not respond to fear in HSF faces. (e) Average activity ( \pm s.e.m.) across conditions for the right thalamic-collicular cluster (mean $x$ y $z, 9-22-7 ; 25$ voxels at $P<0.01$ ) shown in c revealing a pattern similar to the amygdala, with an increased response to fearful faces only for BSF and LSF stimuli.

for neutral faces, $\left.F_{1,11}=542, P<0.0001\right)$ without a main effect of spatial frequency content $\left(F_{2,22}=1.58, P=0.23\right)$, but with a significant interaction of these two factors $\left(F_{2,22}=6.43, P=0.006\right)$. This was due to a slightly higher rating of fearful expressions in BSF (intact) and HSF faces (3.5 and 3.4, respectively) than in LSF faces (3.2; paired $t$ tests, $P<0.05)$, whereas ratings for intact BSF and HSF did not differ (paired $t$-test, $P=0.38$ ). Thus, even though LSF faces produced stronger fear responses in the amygdala under 'incidental' processing (that is, for the gender judgment where fear was task-irrelevant), explicit ratings of fear actually increased slightly with the presence of HSF cues. This may be consistent with a greater role of the HSF range for detailed conscious analysis mediated by ventral visual cortex.

\section{DISCUSSION}

Our results show a dissociation between neural responses in amygdala and extrastriate visual cortex, across different spatial frequency ranges for face stimuli. Whereas fusiform cortex seemed to encode and maintain a representation of faces that was influenced more (though not exclusively) by fine-grained HSF information, the amygdala response to fearful expression was selectively driven by coarse LSF cues. This provides evidence that visual inputs to the amygdala may be partly distinct from those to ventral extrastriate visual cortex. Thus, the amygdala and cortical systems might operate on different subsets of visual information that are both available simultaneously in naturalistic (BSF) images of faces.

First, we found that fusiform cortex, thought to be critically involved in face detection and identification ${ }^{4,25}$, shows a predominant influence of HSF visual information, as it is more activated by BSF and HSF faces overall than by LSF faces. This is consistent with major parvocellular inputs to the ventral visual stream ${ }^{9,10}$ and accords with an important role of HSF information for fine visual shape and texture analysis ${ }^{20,22,23}$. This also provides new neurobiological evidence in support of previous proposals, based solely on behavioral findings, that face recognition in humans may emphasize a range of mid to higher SFs ( $\geq 8-16$ cycles/face), especially for identification tasks ${ }^{18,20,21}$. By contrast, LSF face stimuli evoked much less activity in fusiform cortex, even though some LSF information can reach ventral cortex ${ }^{10}$. Such LSF cues might support perception of face configuration (for example, see Fig. 1) but provide only poor information for detailed identification. However, recognition of identity may still be possible based on LSF information alone when just a small set of previously learned faces is used ${ }^{16,17}$. Higher spatial frequency cues convey richer edge and surface details that may improve identification when finer individual discriminations are required ${ }^{33,34}$ and when different views of the same face are used ${ }^{18,34}$. 
Table 3 Effects of fearful expression

\begin{tabular}{|c|c|c|c|c|c|c|}
\hline \multicolumn{2}{|c|}{ Side Area } & \multicolumn{5}{|c|}{ All fearful > neutral faces (collapsed across all spatial frequency formats) } \\
\hline \multirow[t]{2}{*}{$L$} & fusiform gyrus (ant.) & -36 & -38 & -28 & 6.79 & \\
\hline & (post.) & 40 & -52 & -30 & 3.84 & \\
\hline $\mathrm{R}$ & amygdala & 20 & -10 & -30 & 4.25 & \\
\hline \multirow[t]{2}{*}{ L } & amygdala & -20 & -10 & -28 & 3.91 & \\
\hline & & -24 & -6 & -30 & 2.82 & ** \\
\hline $\mathrm{R}$ & temporal pole & 44 & 8 & -22 & 5.91 & \\
\hline L & superior temporal sulcus & -46 & -4 & -18 & 4.46 & \\
\hline L & anterior occipital sulcus & -40 & -56 & 0 & 4.04 & \\
\hline \multirow[t]{2}{*}{ L } & cingulate gyrus (mid.) & -16 & -6 & 40 & 5.51 & \\
\hline & (post.) & -16 & -34 & 50 & 4.71 & \\
\hline $\mathrm{R}$ & retrosplenial cortex & 10 & -46 & 12 & 4.14 & \\
\hline $\mathrm{R}$ & superior frontal gyrus & 12 & 40 & 52 & 3.81 & \\
\hline \multicolumn{7}{|c|}{ Expression $\times$ frequency interaction (response to fearful LSF $>$ HSF faces) } \\
\hline \multirow[t]{2}{*}{$\mathrm{L}$} & amygdala & -22 & 4 & -18 & 4.07 & \\
\hline & & -16 & -8 & -18 & 3.57 & * \\
\hline \multirow[t]{2}{*}{$\mathrm{R}$} & amygdala & 18 & 2 & -20 & 3.35 & * \\
\hline & & 22 & 0 & -30 & 3.05 & * \\
\hline L & $\begin{array}{l}\text { posterior thalamus } \\
\text { (lateral) }\end{array}$ & -16 & -18 & 0 & 4.64 & \\
\hline \multirow[t]{2}{*}{$\mathrm{R}$} & $\begin{array}{l}\text { posterior thalamus } \\
\text { (lateral) }\end{array}$ & 18 & -16 & 6 & 4.97 & \\
\hline & (inferior) & 10 & -20 & -8 & 5.9 & \\
\hline $\mathrm{R}$ & superior colliculus & 2 & -30 & -18 & 3.81 & \\
\hline \multirow[t]{2}{*}{ L } & posterior insula & -36 & -22 & 18 & 4.71 & \\
\hline & & -38 & -32 & 20 & 4.25 & \\
\hline \multirow[t]{2}{*}{$\mathrm{R}$} & anterior insula & 30 & 10 & -12 & 5.31 & \\
\hline & & 34 & 12 & 0 & 4.66 & \\
\hline L & retrosplenial cortex & -10 & -52 & 6 & 7.25 & \\
\hline $\mathrm{R}$ & retrosplenial cortex & 12 & -44 & 20 & 4.46 & \\
\hline $\mathrm{R}$ & posterior cingulate & 8 & -54 & 46 & 3.82 & \\
\hline $\mathrm{R}$ & medial parietal cortex & 8 & -34 & 66 & 5.81 & \\
\hline $\mathrm{R}$ & intraparietal sulcus & 36 & -62 & 32 & 5.3 & \\
\hline $\mathrm{R}$ & temporo-occipital cortex & 58 & -54 & 18 & 3.96 & \\
\hline $\mathrm{L}$ & premotor cortex & -54 & -8 & 32 & 4.08 & \\
\hline $\mathrm{R}$ & pons & 14 & -28 & -30 & 4.92 & \\
\hline
\end{tabular}

All peaks $P<0.001$ uncorrected (random-effect analysis), except ${ }^{*} P<0.005,{ }^{*} P<0.01$.

Consistent with this, we also found that only HSF stimuli established a lasting representation in fusiform cortex that could influence subsequent processing, so as to produce repetition-priming decreases in the fMRI signal when a previously exposed HSF face was subsequently shown in a different, impoverished (LSF) image. LSF stimuli are apparently unable to lay down durable traces that can influence subsequent recognition of related images (across a change in bandpass) in an analogous way. Moreover, this generalization across different face images (from HSF to LSF) implicated not only posterior but also more anterior fusiform regions, where the representation of face identity may be more abstract from physical properties ${ }^{29}$. These anterior fusiform regions may code for invariant structural face identity, rather than simple featural traits ${ }^{24,29,34}$

In contrast to activity in occipito-temporal cortices being enhanced by HSF information for faces, neural responses to fearful expressions in amygdala and interconnected subcortical areas were driven by LSF visual information. The amygdala is comparatively 'blind' to fearful expression in HSF faces, even though HSF features produce optimal activation of fusiform cortex and carry rich visual details about facial wrinkles that can be associated with specific emotional expressions. Given extensive interconnections between the amygdala and the ventral stream, a lack of amygdala responses to HSF driving fusiform areas may suggest that face-sensitive processes in fusiform do not carry information about expression per se, but rather carry invariant structural information necessary for identification ${ }^{4,25,35}$. Other changeable aspects of faces might implicate distinct regions, with the amygdala being more sensitive to emotion apparently extracted from crude LSF cues, as shown here. These differential amygdala responses to fear as a function of spatial frequency during 'incidental' processing (during gender judgments, when expression was irrelevant) cannot be explained by a better explicit perception of expression in LSF faces, as demonstrated by our behavioral rating study. These data might also be taken to suggest that conscious ratings of fearful expression may not depend exclusively on amygdala activity, in keeping with varied findings on explicit fear recognition after amygdala lesions in humans ${ }^{36,37}$. HSF features that are ineffective in driving amygdala responses might still allow detailed conscious judgments mediated by extrastriate cortical processing. Further studies may address whether cortical processing of HSF aspects of emotional expression can drive the amygdala during tasks involving explicit judgments of expression, rather than the incidental task used here.

As in the amygdala, we also found selective responses to LSF fearful faces in the thalamus and superior colliculus. These subcortical regions showed a significant interaction between emotional expression and spatial frequency, indicating an activation by fearful faces specifically for LSF as compared with HSF inputs. This is consistent with the idea that tecto-pulvinar pathways may provide the amygdala with coarse (LSF) visual signals about fear-related stimuli. Our thalamic foci fit well with the lateral and inferior visual subsections in anterior pulvinar that receive major afferents from superior colliculus via a ventral fiber tract ${ }^{31,38,39}$. Pulvinar nuclei may also receive direct retinal inputs ${ }^{40,41}$, and in turn send monosynaptic projections to the amygdala ${ }^{31,42,43}$, as well as to higher-level areas in temporal, posterior cingulate and orbitofrontal cortex ${ }^{31}$. A selective engagement of these tecto-pulvinar pathways by the LSF range also fits with their receiving inputs of a magnocellular type ${ }^{13,44,45}$ and with the low-pass tuning characteristics of residual 'blindsight' mediated by these pathways after cortical visual damage ${ }^{46}$.

The idea that phylogenetically old subcortical pathways are involved in conveying LSF visual information to a dedicated dangerdetection system centered on the amygdala ${ }^{1}$ has several wider implications. Faster processing of visual inputs within magnocellular than parvocellular pathways ${ }^{9,10}$ could aid speedy, survival-enhancing responses to danger. It might also explain otherwise paradoxical electrophysiological findings that neural responses to fearful face expressions ( $\sim 120 \mathrm{~ms}$ after onset) can precede face-specific responses from ventral visual cortex (at $\sim 170 \mathrm{~ms})^{47,48}$. LSF information in magnocellular channels is crucial for processing stimuli in the visual periphery ${ }^{9}$, at a distance ${ }^{49}$ or in motion ${ }^{10}$, which seems advantageous for detection of potential danger. LSF information also underlies most visual abilities of newborn infants, who can detect coarse facial and emotional cues in the absence of a mature cortical visual system ${ }^{50}$.

In summary, our results reveal a distinct pattern of spatial frequency sensitivities in the human brain for processing faces and their emotional fearful expressions. Whereas HSF information in faces travels via parvocellular inputs into ventral cortical pathways and 
evokes responses in fusiform cortex, responses in amygdala and subcortical areas are greater for LSF cues carried primarily by magnocellular visual pathways. Our findings suggest that subcortical tecto-pulvinar pathways may provide the amygdala with coarse, but rapid, fear-related information based on LSF features, independent of slower conscious analysis based on HSF in cortical pathways ${ }^{1,5,6}$. These distinct response properties delineate segregated anatomical routes for facial and emotional processing. Moreover, they provide new insights into the different nature of face representations in fusiform cortex and amygdala, in a manner that relates to basic neurophysiological visual channels ${ }^{9,10}$.

\section{METHODS}

Subjects. Participants were 13 healthy volunteers in the imaging experiment ( 7 females; age range 18-37, mean 27) and 12 healthy volunteers in the behavioral control experiment ( 6 females; age 18-40, mean 29). All were righthanded, had normal or corrected vision, no past neurological or psychiatric history and no structural brain abnormality. Informed consent was obtained according to procedures approved by the Joint Ethics Committee of the National Hospital and Institute of Neurology, London.

Stimuli. Stimuli were black-and-white photographs of emotional and neutral faces, taken from the Karolinska Directed Emotional Faces set (KDEF, Lundqvist, D., Flykt, A. \& Ohman, A.; Dept. of Neurosciences, Karolinska Hospital, Stockholm, Sweden, 1998) and our own set (unpublished). All faces were enclosed in a rectangular frame excluding most of the hair and non-facial contours (Fig. 1), in a $198 \times 252$ pixel array, back-projected onto a mirror mounted on the MRI head coil (visual angle $\sim 5 \times 6.3^{\circ}$ ). Spatial frequency content in the original stimuli (broad-band, BSF) was filtered using a high-pass cut-off that was $>24$ cycles/image for the HSF stimuli, and a low-pass cut-off of $<6$ cycles/image for the LSF stimuli. Average stimulus luminance did not differ according to image type (mean across all stimuli used for BSF, HSF and LSF faces: 112,118 and 115 on a 256 gray-level scale; $F_{1,547}=1.32, P=0.27$ ); neither did it differ between neutral and fearful faces (mean, 117 and 114, respectively; $F_{1,548}<0.01, P=0.99$ ). Each face was shown centrally for $200 \mathrm{~ms}$, with a mean interstimulus interval of $6.1 \mathrm{~s}$, and onset jittered with respect to scan repetition times (TRs). All different types of stimuli were shown in random order, with the face of a given individual person shown only twice (first exposure followed by repetition in either the same format (for BSF images) or different image format (for HSF or LSF images). Only BSF faces were repeated in the same format, whereas faces presented initially in HSF were later repeated in LSF, or vice versa, such that each filtered face was primed only by the other filtered version, but never preceded nor followed by a complete BSF version. Individual faces were counterbalanced (with different expressions and different spatial frequency contents) across participants.

Scanning and data analysis. Whole-brain fMRI data were acquired on a 2T Magnetom VISION system (Siemens) equipped with a head volume coil. Functional images were obtained with a gradient echo-planar $\mathrm{T} 2{ }^{*}$ sequence using blood oxygenation level-dependent (BOLD) contrast, each comprising a full brain volume of 32 contiguous axial slices ( $3 \mathrm{~mm}$ thickness). Volumes were acquired continuously with a repetition time (TR) of $3.17 \mathrm{~s}$. A total of 482 scans were acquired for each participant in a single session (25 min), with the first eight volumes subsequently discarded to allow for T1 equilibration effects.

Functional MRI data were analyzed using the general linear model for event-related designs in SPM99 (Wellcome Dept. of Imaging Neuroscience; www.fil.ion.ucl.ac.uk/spm). Individual scans were realigned, normalized, time-corrected and spatially smoothed by an 8-mm FWHM Gaussian kernel using standard SPM methods. A high-pass frequency filter (cut-off $120 \mathrm{~s}$ ) and corrections for auto-correlation between scans were applied to the time series. Each event was modeled by a standard synthetic hemodynamic response function at each voxel across the whole brain, and movement parameters from realignment corrections were entered as covariates of no interest. Parameter estimates of event-related activity were obtained at each voxel, for each condition and each subject. Statistical parametric maps of the $t$-statistic ( $\operatorname{SPM}\{t\})$ were generated from linear contrasts between different conditions and transformed to a normal distribution $(\operatorname{SPM}\{Z\})$ for each individual participant. A two-stage random-effect analysis was then performed using a one-sample $t$-test on contrast images obtained in each subject for each comparison of interest, treating subjects as a random variable (after additional 7-mm smoothing to account for inter-individual variability, according to usual random-effect methods). In this random-effect analysis, resulting SPMs of the $t$-statistic (d.f. $=12$ ) at each voxel were thresholded at $P<0.001$ uncorrected. Additional activations at lower thresholds are reported for descriptive purposes when consistent with prior hypotheses and other reported results.

During fMRI scanning, eye position was monitored on-line by an infrared eye tracker (ASL Model 504, Applied Science Group) and subsequently analyzed off-line to compute mean eye-position changes along the vertical and horizontal axes during a 200 -ms period after stimulus onset, relative to $200 \mathrm{~ms}$ before, for each trial and each subject. These data were analyzed by a 3 (spatial frequency) $\times 2$ (facial expression) $\times 2$ (identity repetition) repeated-measure ANOVA.

Note: Supplementary information is available on the Nature Neuroscience website.

\section{ACKNOWLEDGMENTS}

Supported by Wellcome Programme grants (R.J.D. and J.D.), an MRC (UK) cooperative grant at University College London and a Swiss National Science Foundation grant (P.V.). J.D. holds a Royal Society-Wolfson Research Merit Award.

\section{COMPETING INTERESTS STATEMENT}

The authors declare that they have no competing financial interests.

Received 30 September 2002; accepted 1 April 2003

Published online 12 May 2003; doi:10.1038/nn1057

1. LeDoux, J.E. The Emotional Brain (Simon \& Schuster, New York, 1996).

2. Morris, J., Öhman, A. \& Dolan, R.J. Conscious and unconscious emotional learning in the human amygdala. Nature 393, 467-470 (1998).

3. Whalen, P.J. et al. Masked presentations of emotional facial expressions modulate amygdala activity without explicit knowledge. J. Neurosci. 18, 411-418 (1998).

4. Vuilleumier, P., Armony, J.L., Driver, J. \& Dolan, R.J. Effects of attention and emotion on face processing in the human brain: an event-related fMRI study. Neuron 30, 829-841 (2001)

5. Morris, J.S., Öhman, A. \& Dolan, R.J. A subcortical pathway to the right amygdala mediating 'unseen' fear. Proc. Natl. Acad. Sci. USA 96, 1680-1685 (1999).

6. de Gelder, B., Vroomen, J., Pourtois, G. \& Weiskrantz, L. Non-conscious recognition of affect in the absence of striate cortex. Neuroreport 10, 3759-3763 (1999).

7. Weiskrantz, L. Blindsight: a Case Study and Implications (Oxford Univ. Press, Oxford, 1986).

8. Morris, J.S., DeGelder, B., Weiskrantz, L. \& Dolan, R.J. Differential extrageniculostriate and amygdala responses to presentation of emotional faces in a cortically blind field. Brain 124, 1241-1252 (2001).

9. Livingstone, M. \& Hubel, D. Segregation of form, color, movement and depth: anatomy, physiology and perception. Science 240, 740-749 (1988).

10. Merigan, W.H. \& Maunsell, J.H. How parallel are the primate visual pathways? Annu. Rev. Neurosci. 16, 369-402 (1993).

11. Schiller, P.H., Malpeli, J.G. \& Schein, S.J. Composition of geniculostriate input to superior colliculus of the rhesus monkey. J. Neurophysiol. 42, 1124-1133 (1979).

12. Leventhal, A.G., Rodieck, R.W. \& Dreher, B. Central projections of cat retinal ganglion cells. J. Comp. Neurol. 237, 216-226 (1985).

13. Berson, D.M. Retinal and cortical inputs to cat superior colliculus: composition, convergence and laminar specificity. Prog. Brain Res. 75, 17-26 (1988).

14. Sergent, J. Influence of task and input factors on hemispheric involvement in face processing. J. Exp. Psychol. Hum. Percept. Perform. 11, 846-861 (1985).

15. Costen, N.P., Parker, D.M. \& Craw, I. Effects of high-pass and low-pass spatial filtering on face identification. Percept. Psychophys. 58, 602-612 (1996).

16. Schyns, P.G. \& Oliva, A. Dr. Angry and Mr. Smile: when categorization flexibly modifies the perception of faces in rapid visual presentations. Cognition 69, 243-265 (1999)

17. Nasanen, R. Spatial frequency bandwidth used in the recognition of facial images. Vision Res. 39, 3824-3833 (1999).

18. Liu, C.H., Collin, C.A., Rainville, S.J. \& Chaudhuri, A. The effects of spatial frequency overlap on face recognition. J. Exp. Psychol. Hum. Percept. Perform. 26, 956-979 (2000)

19. Calder, A.J., Young, A.W., Keane, J. \& Dean, M. Configural information in facial expression perception. J. Exp. Psychol. Hum. Percept. Perform. 26, 527-551 (2000). 
20. Tieger, T. \& Ganz, L. Recognition of faces in the presence of two-dimensional sinusoidal masks. Percept. Psychophys. 26, 163-167 (1979).

21. Fiorentini, A., Maffei, L. \& Sandini, G. The role of high spatial frequencies in face perception. Perception 12, 195-201 (1983)

22. Hayes, T., Morrone, M.C. \& Burr, D.C. Recognition of positive and negative bandpass-filtered images. Perception 15, 595-602 (1986).

23. Norman, J. \& Ehrlich, S. Spatial frequency filtering and target identification. Vision Res. 27, 87-96 (1987).

24. Goshen-Gottstein, Y. \& Ganel, T. Repetition priming for familiar and unfamilia faces in a sex-judgment task: evidence for a common route for the processing of sex and identity. J. Exp. Psychol. Learn. Mem. Cogn. 26, 1198-1214 (2000).

25. Kanwisher, N., McDermott, J. \& Chun, M.M. The fusiform face area: a module in human extrastriate cortex specialized for face perception. J. Neurosci. 17, 4302-4311 (1997)

26. Naccache, L. \& Dehaene, S. The priming method: imaging unconscious repetition priming reveals an abstract representation of number in the parietal lobes. Cereb. Cortex 11, 966-974 (2001).

27. Henson, R., Shallice, T. \& Dolan, R.J. Neuroimaging evidence for dissociable forms of repetition priming. Science 287, 1269-1272 (2000)

28. Burton, A.M., Miller, P., Bruce, V., Hancock, P.J. \& Henderson, Z. Human and automatic face recognition: a comparison across image formats. Vision Res. $\mathbf{4 1}$ 3185-3195 (2001)

29. George, N. et al. Contrast polarity and face recognition in the human fusiform gyrus. Nat. Neurosci. 2, 574-580 (1999).

30. Phan, K.L., Wager, T., Taylor, S.F. \& Liberzon, I. Functional neuroanatomy of emotion: a meta-analysis of emotion activation studies in PET and fMRI. Neuroimage 16, 331-348 (2002).

31. Robinson, D.L. \& Petersen, S.E. The pulvinar and visual salience. Trends Neurosci. 15, 127-132 (1992)

32. Morris, J.S. et al. A neuromodulatory role for the human amygdala in processing emotional facial expressions. J. Comp. Neurol. 394, 91-105 (1998).

33. Bruce, V. et al. Recognising facial surfaces. Perception 20, 755-769 (1991).

34. Hill, H., Schyns, P.G. \& Akamatsu, S. Information and viewpoint dependence in face recognition. Cognition 62, 201-222 (1997).

35. Haxby, J.V., Hoffman, E.A. \& Gobbini, M.I. The distributed human neural system for face perception. Trends Cogn. Sci. 4, 223-233 (2000).

36. Adolphs, R. et al. Recognition of facial emotion in nine individuals with bilatera amygdala damage. Neuropsychologia 37, 1111-1117 (1999).
37. Hamann, S.B. \& Adolphs, R. Normal recognition of emotional similarity between facial expressions following bilateral amygdala damage. Neuropsychologia 37 1135-1141 (1999).

38. Harting, J.K., Huerta, M.F., Frankfurter, A.J., Strominger, N.L. \& Royce, G.J. Ascending pathways from the monkey superior colliculus: an autoradiographic analysis. J. Comp. Neurol. 192, 853-882 (1980).

39 Benevento, L.A. \& Standage, G.P. The organization of projections of the retinorecipient and nonretinorecipient nuclei of the pretectal complex and layers of the superior colliculus to the lateral pulvinar and medial pulvinar in the macaque monkey. J. Comp. Neurol. 217, 307-336 (1983).

40. O'Brien, B.J., Abel, P.L. \& Olavarria, J.F. The retinal input to calbindin-D28kdefined subdivisions in macaque inferior pulvinar. Neurosci. Lett. 312, $145-148$ (2001).

41. Cowey, A., Stoerig, P. \& Bannister, M. Retinal ganglion cells labeled from the pulvinar nucleus in macaque monkeys. Neuroscience 61, 691-705 (1994).

42. Jones, E.G. \& Burton, H. A projection from the medial pulvinar to the amygdala in primates. Brain Res. 104, 142-147 (1976).

43. Linke, R., Braune, G. \& Schwegler, H. Differential projection of the posterior paralaminar thalamic nuclei to the amygdaloid complex in the rat. Exp. Brain Res. 134, 520-532 (2000).

44. Bisti, S. \& Sireteanu, R.C. Sensitivity to spatial frequency and contrast of visual cells in the cat superior colliculus. Vision Res. 16, 247-251 (1976).

45. Lomber, S.G. Learning to see the trees before the forest: reversible deactivation of the superior colliculus during learning of local and global visual features. Proc. Natl. Acad. Sci. USA 99, 4049-4054 (2002)

46. Sahraie, A., Weiskrantz, L., Trevethan, C.T., Cruce, R. \& Murray, A.D. Psychophysical and pupillometric study of spatial channels of visual processing in blindsight. Exp. Brain Res. 143, 249-256 (2002).

47. Kawasaki, H. et al. Single-neuron responses to emotional visual stimuli recorded in human ventral prefrontal cortex. Nat. Neurosci. 4, 15-16 (2001).

48. Eimer, M. \& Holmes, A. An ERP study on the time course of emotional face processing. Neuroreport 13, 427-431 (2002).

49. Kline, D.W. \& Fuchs, P. The visibility of symbolic highway signs can be increased among drivers of all ages. Hum. Factors 35, 25-34 (1993)

50. Johnson, M.H. \& Vecera, S.P. Cortical Parcellation and the Development of Face Processing (eds. de Boysson-Bardies, B., de Shoenen, S., Jusczyk, P.W., McNeilage, P. \& Morton, J.) (Kluwer Academic, Dordrecht, Netherlands, 1993). 